\title{
An incidentally Diagnosed Hepatocellular Carcinoma That Exhibited Unusual Metastasis
}

\author{
Deryem DEMIR, ${ }^{1}$ (D) Tuba TASLAMACIOĞLU DUMAN, ${ }^{1}$ (I) Ümmügül ÜYETÜRK, ${ }^{2}$ \\ (D) Selma ERDOĞAN DÜZCÜ, ${ }^{3}$ (1) Buket Büşra BAŞAR ${ }^{1}$ \\ 'Department of Internal Medicine, Abant Izzet Baysal University Training and Research Hospital, Bolu-Turkey \\ 2Department of Medical Oncology, Abant İzzet Baysal University, Training and Research Hospital, Bolu-Turkey \\ ${ }^{3}$ Department of Pathology, Abant İzzet Baysal University, Training and Research Hospital, Bolu-Turkey
}

\begin{abstract}
SUMMARY
Hepatocellular carcinoma (HCC) is the most common primary liver cancer. It can be diagnosed at a late stage due to the lack of early symptom onset and its non-pathognomonic symptoms. Metastatic regions often include the lung, lymph nodes, bones, and adrenal glands. Soft tissue and central nervous system (CNS) metastases are extremely rare. The incidence of CNS metastasis ranges from $0.6 \%$ to $1.7 \%$. Curative treatment is liver transplantation or resection in early stages. In advanced stages, local therapies include transarterial chemoembolization and radioembolization. Systemic treatments, such as sorafenib, regorafenib, ramucirumab, and nivolumab, are applied in the metastatic stage. We aimed to present with literature the case of HCC that was diagnosed incidentally and had rare metastasis of soft tissue and CNS and long-term overall survival.
\end{abstract}

Keywords: Brain metastasis; hepatocellular carcinoma; incidentally diagnosed.

Copyright $\odot$ 2019, Turkish Society for Radiation Oncology

\section{Introduction}

Hepatocellular carcinoma (HCC) is the most common primary liver cancer and fourth most common cause of cancer-related death; its incidence is increasing.[1] HCC develops after chronic hepatitis B and C infections in approximately $80 \%$ of patients.[2] In addition to viral infections, alcohol consumption, aflatoxin exposure, metabolic disorders, such as hereditary hemochromatosis, alpha-1 antitrypsin deficiency, primary biliary cirrhosis, obesity, diabetes mellitus, and nonalcoholic fatty liver are important in HCC etiology.[3,4]

Most cases of HCC are diagnosed late because of the absence of early and pathognomonic symptoms. Lung, lymph node, bone, and adrenal gland metastases are common; soft tissue and central nervous system (CNS) metastases are extremely rare, and the incidence of CNS metastases ranges from $0.6 \%$ to $1.7 \%$.[5,6] Early stage $\mathrm{HCC}$ is cured via liver transplantation and resection. Advanced stage disease is treated via regional transarterial chemoembolization (TACE) and radioembolization and metastases with first-line sorafenib, followed by secondline regorafenib, ramucirumab, and nivolumab. $[7,8]$

Here, we present a case with incidentally diagnosed unresectable HCC accompanied by rare metastases to soft tissue and the brain and had long-term overall survival.

\section{Case Report}

A 53-year-old male patient complained of breathlessness and coughing only chronic obstructive pulmonary 
disease (COPD) 5 years in his history. He did not drink alcohol or smoke cigarettes and was not infected with hepatitis $\mathrm{B}$ or $\mathrm{C}$ virus. There was no property in his family history. Thorax computed tomography examination was performed because of COPD symptoms. Solid lesions were detected in the liver segment 4A-8 junction on December 18, 2013. Three similar solid tumors were evident on upper abdominal magnetic resonance imaging (MRI); the largest was in segment 8 with $47 \times 55 \times 37 \mathrm{~mm}$ in size, and the others were in segments $6-7$. The alpha-fetoprotein (AFP) level was 2.35 (normal range: $0-7$ ) $\mathrm{ng} / \mathrm{mL}$.

Neither gastroscopy nor colonoscopy yielded any indication of malignancy. A tru-cut liver biopsy revealed morphologically and immunohistochemically well-differentiated HCC. TACE was performed using Adriamycin. The liver was considered non-resectable given the number of lesions and their multiple locations. Tumor regression (40\%) was evident after TACE. During follow-up, the AFP level increased to $25.1 \mathrm{ng} / \mathrm{mL}$, and abdominal MRI revealed new liver lesions. The ChildPugh score was A (5). TACE with Adriamycin was repeated. On June 6, 2016, thoracic MRI was repeated because the parasternum was stiff. A nodular metastatic lesion was evident in the parasternal area accompanied by posterior peripheral contrast. A biopsy revealed HCC metastasis (Fig. 1). Sorafenib was started $400 \mathrm{mg}$ /day. $[9,10]$ After 5 months, capecitabine chemotherapy was started when soft tissue metastasis progressed.[11]

Four months after that, the patient developed speech and perceptual disorders, and brain MRI revealed a right frontal metastasis (Fig. 2). An operation followed. The pathologist diagnosed a malignant tumor composed of pleomorphic nucleated cells with

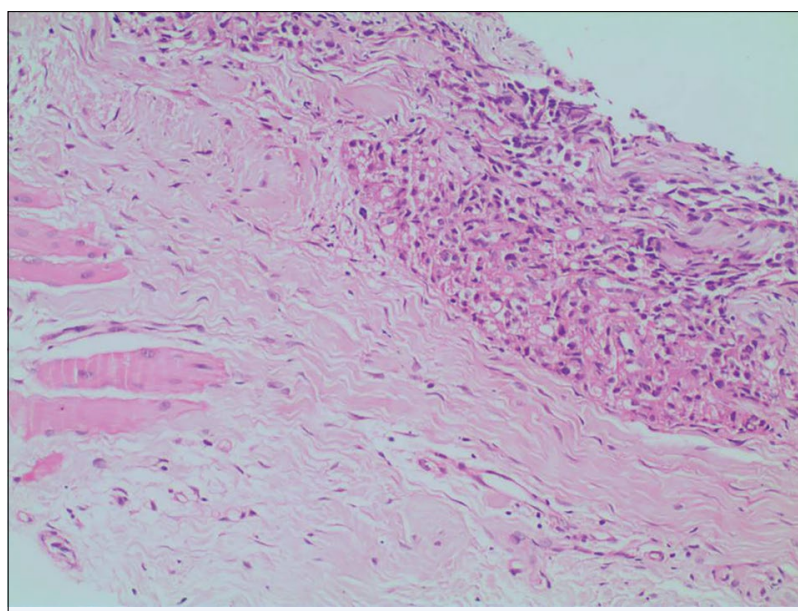

Fig. 1. Metastatic tumor tissue near striated muscle tissue in fibrous tissue, HEX200.

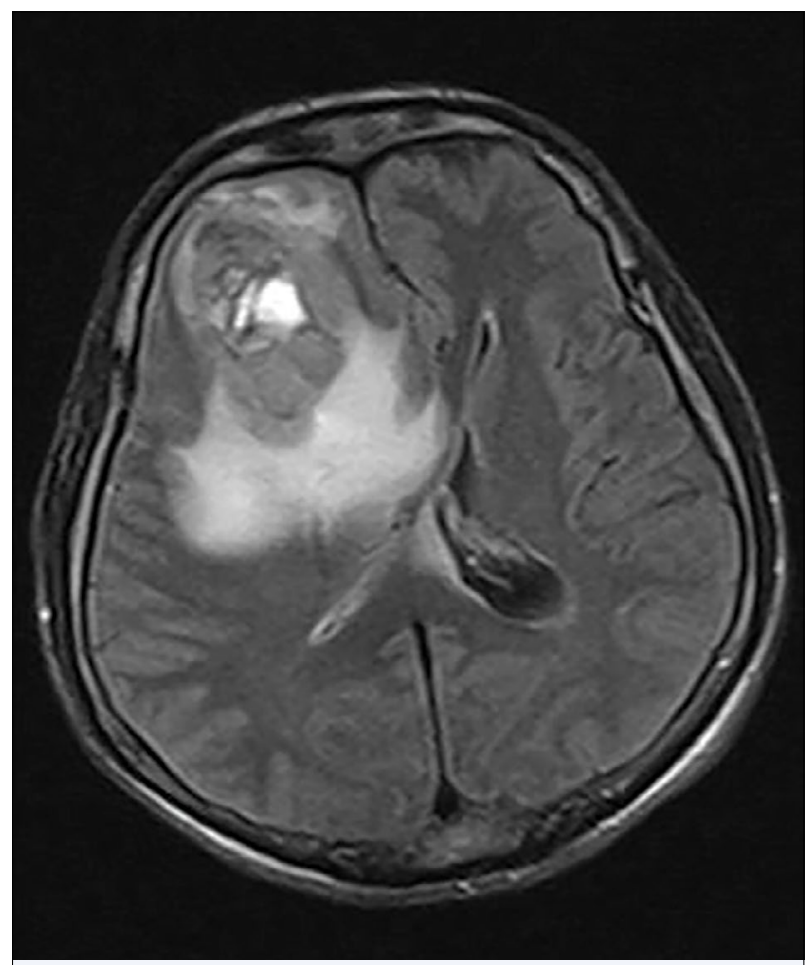

Fig. 2. Brain Magnetic Resonance Imaging: Metastatic lesion with a heterogeneous nature, which has a wide hyperintense area in the right frontal lobe anterior, causing right lateral ventricule and left lateral ventricule anterior horn compression and midline shift.

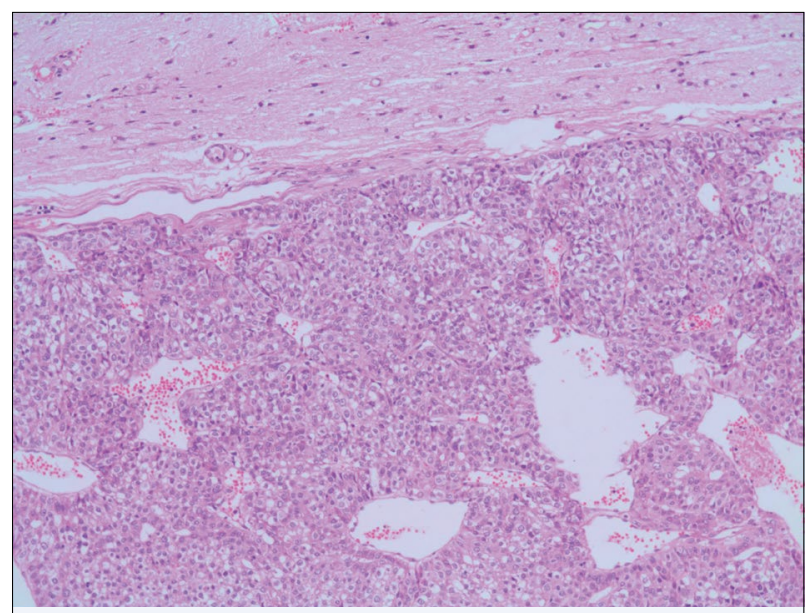

Fig. 3. Tumor tissue forming acinar structures adjacent to glial tissue, HEX100.

eosinophilic cytoplasm that formed acinar structures. The cells stained positively for cytokeratin (CK)-7 and focally for hepatocyte paraffin-1, but not for glial fibrillary acidic protein, CK-20, or CDX2, thus indicating HCC metastasis (Fig. 3). Palliative radiotherapy was 
performed, and the patient was started on regorafenib (160 mg daily).[12]

Palliative treatment continued for 3 months associated with disease progression. The patient died on December 25, 2017 after 48 months of follow-up.

\section{Discussion}

HCC can be long-term asymptomatic prior to diagnosis, and surgical resection is impossible in many cases. Although our case was incidentally diagnosed, the lesions were both central and bilobular such that surgery was not an option. Local treatments include TACE and radioembolization; these trigger a tumor response and prolong survival.[13,14] Combined chemoembolization, external radiotherapy, or localized liver ablation are better than embolization alone, prolonging the median survival time by up to 20 months. $[13,15]$ Chemoembolization was performed employing Adriamycin.

Although HCCs featuring a high tumor burden and/or adjacent organ infiltration/metastasis are often treated with sorafenib, one study compared 172 patients treated via TACE and sorafenib, and the median survival times were 8 and 7 months, respectively $(p=0.312)$. It was concluded that TACE was not inferior to sorafenib, especially in patients with limited tumor spread.[16] The present case initially showed no metastasis or adjacent organ infiltration, but TACE was indicated by the high liver tumor burden.

HCC is a hypervascular tumor that usually spreads hematogenously to the lungs, lymph nodes, and bones. [10] Metastatic HCC behavior can now be altered by new treatments. In some cases, metastases are found in unusual areas.[17] In one study, soft tissue metastases were detected in only 3 out of 32 patients, and the most common metastatic sites continued to be the bones and lungs.[18] Our case exhibited only soft tissue metastasis.

Systemic therapies are indicated for HCC metastasis. The tyrosine kinase inhibitor sorafenib is used to treat advanced HCC cases that do not respond to TACE. The SHARP study compared sorafenib and placebo after TACE. The median radiological progression times were 5.5 months in the sorafenib group and 2.8 months in the placebo group $(\mathrm{p}<0.001)$. [9] In a study in the Asia-Pacific region, the median survival times were 6.5 months in the sorafenib group and 4.2 months in the placebo group ( $\mathrm{p}=0.014)$. [10] The patient was started on sorafenib at 30 months after TACE when an isolated soft tissue metastasis was apparent and the liver lesions had progressed.
Capecitabine chemotherapy was later instituted. In a study comparing capecitabine with best-supportive care (BSC), the median overall survival times were 12.0 (95\% confidence interval (CI) 10.7-15.8) months in patients receiving capecitabine and 9.0 (95\% CI 6.5-13.9) months in those under BSC. Capecitabine was a safe second-line treatment for patients with HCC.[11]

The frequency of HCC brain metastases is increasing because new treatments are prolonging patient survival. Brain metastasis usually manifests as intracerebral hemorrhages.[19] In a 9-year retrospective study of patients with HCC in Tokyo, 19 developed brain metastases at a median of 32 months after diagnosis, and the median survival time was 21 weeks after metastasis diagnosis. Brain metastasis was associated with a poor performance score and poor prognosis. [20] In a study of 592 patients with advanced stage HCC, brain metastases developed in $8(1.3 \%)$. Particularly in patients with lung metastases, high levels of tumor markers in the prior 3-6 months predicted the development of brain metastases. [21] Our patient developed brain metastasis at 11 months after soft tissue metastasis, and the AFP level increased 4000-fold during this interval. Multidisciplinary approaches, such as surgery, whole brain irradiation, and stereotactic radiosurgery, are required for patients with brain metastases.[19] Our patient underwent both surgery and radiotherapy.

Ramucirumab, nivolumab, and regorafenib are second-line treatments for metastatic HCC. Vascular endothelial growth factor (VEGF) expression and VEGF receptor-2-associated angiogenesis feature prominently in HCC pathogenesis. Ramucirumab is a recombinant IgG1 monoclonal antibody recognizing VEGF receptor-2. The randomized, multicenter, Phase $3 \mathrm{REACH}$ trial study administered ramucirumab $8 \mathrm{mg} /$ $\mathrm{kg}$ daily or placebo to patients evidencing sorafenib intolerance or disease progression. The median survival times were 9.2 and 7.6 months, respectively $(\mathrm{p}=0.14)$. Thus, the second-line treatment was no better than placebo.[22]

Nivolumab is a human IgG4-kappa monoclonal antibody inhibiting the interaction of PD-L1 and PD-L2 [7,8], thus promoting programmed cell death. The phase $1 / 2$ CheckMate 040 study of patients with advanced HCC with or without chronic viral hepatitis showed that nivolumab was both effective and safe.[23]

Regorafenib is a tyrosine kinase inhibitor targeting the immunoglobulin-like domain, epidermal growth factor-like domain 2, fibroblast growth factor receptor, c-kit, Ret, VEGF, platelet-derived growth factor 
receptor, and rapidly accelerated fibrosarcoma-mitogen-activated protein kinase-extracellular-regulated kinase. The randomized, international, multicenter, phase III RESORCE study evaluated the post-sorafenib efficacy of regorafenib. The drug increased the median survival of post-sorafenib patients from 7.8 months to 10.6 months. [12] Second-line capecitabine was administered because regorafenib was not approved by the Food and Drug Administration on April 27, 2017. Regorafenib was later prescribed. Progression was evident after 3 months, and the drug was terminated. Palliative care (only) followed; the patient died 48 months after the initial diagnosis.

\section{Conclusion}

Unusual regional metastases are becoming more common in patients with HCC as survival is prolonged. Multidisciplinary approaches are increasingly important when treating HCC. Survival is prolonged by surgery, local treatments, and systemic sorafenib, regorafenib, ramucirumab, and nivolumab.

Informed consent: Written informed consent was obtained from the patient for the publication of the case report and the accompanying images.

Peer-review: Externally peer-reviewed.

Conflict of Interest: The authors have no conflict of interests to declare.

Financial Disclosure: The authors declared that this study has received no financial support.

Authorship contributions: Concept - M.D., T.T.D., Ü.Ü.; Design - T.T.D., Ü.Ü.; Supervision - T.T.D., Ü.Ü.; Materials - S.E.D., B.B.B.; Data collection \&/or processing - M.D., S.E.D., B.B.B.; Analysis and/or interpretation - M.D., T.T.D., Ü.Ü.; Literature search - M.D., T.T.D., Ü.Ü.; Writing - M.D., T.T.D., Ü.Ü.; Critical review - T.D.T., Ü.Ü.

\section{References}

1. Ferlay J, Colombet M, Soerjomataram I, Mathers C, Parkin DM, Piñeros M, et al. Estimating the global cancer incidence and mortality in 2018: GLOBOCAN sourcesand methods. Int J Cancer 2019;144(8):194153.

2. Chung JW, Jang ES, Kim J, Jeong SH, Kim N, Lee DH, et al. Development of a nomogram for screening of hepatitis B virus-associated hepatocellular carcinoma. Oncotarget 2017;8(63):106499-510.

3. Makarova-Rusher OV, Altekruse SF, McNeel TS, Ulahannan S, Duffy AG, Graubard BI, et al. Population attributable fractions of risk factors for hepatocellular carcinoma in the United States. Cancer 2016;122(11):1757-65.
4. Fattovich G, Stroffolini T, Zagni I, Donato F. Hepatocellular carcinoma in cirrhosis: incidence and risk factors. Gastroenterology 2004;127(5 Suppl 1):S35-50.

5. Motola-Kuba D, Zamora-Valdés D, Uribe M, Méndez-Sánchez N. Hepatocellular carcinoma. An overview. Ann Hepatol 2006;5(1):16-24.

6. Sartori Balbinot R, Facco Muscope AL, Dal Castel M, Sartori Balbinot S, Angelo Balbinot R, Soldera J. Intraparenchymal Hemorrhage due to Brain Metastasis of Hepatocellular Carcinoma. Case Rep Gastroenterol 2017;11(3):516-25.

7. Rinninella E, Cerrito L, Spinelli I, Cintoni M, Mele MC, Pompili M, et al. Chemotherapy for Hepatocellular Carcinoma: Current Evidence and FuturePerspectives. J Clin Transl Hepatol 2017;5(3):235-48.

8. Daher S, Massarwa M, Benson AA, Khoury T. Current and Future Treatment of Hepatocellular Carcinoma: An Updated Comprehensive Review. J Clin Transl Hepatol 2018;6(1):69-78.

9. Llovet JM, Ricci S, Mazzaferro V, Hilgard P, Gane E, Blanc JF, et al.; SHARP Investigators Study Group. Sorafenib in advanced hepatocellular carcinoma. $\mathrm{N}$ Engl J Med 2008;359(4):378-90.

10. Cheng AL, Kang YK, Chen Z, Tsao CJ, Qin S, Kim JS, et al. Efficacy and safety of sorafenib in patients in the Asia-Pacific region with advanced hepatocellular carcinoma: a phase III randomised, double-blind, placebo-controlled trial. Lancet Oncol 2009;10(1):25-34.

11. Casadei Gardini A, Foca F, Scartozzi M, Silvestris N, Tamburini E, Faloppi L, et al. Metronomic capecitabine versus best supportive care as secondline treatment in hepatocellular carcinoma: a retrospective study. Sci Rep 2017;7:42499.

12. Bruix J, Qin S, Merle P, Granito A, Huang YH, Bodoky $\mathrm{G}$, et al.; RESORCE Investigators. Regorafenib for patients with hepatocellular carcinoma who progressed on sorafenib treatment (RESORCE): a randomised, double-blind, placebo-controlled, phase 3 trial. Lancet 2017;389(10064):56-66.

13. Oliveri RS, Wetterslev J, Gluud C. Transarterial (chemo)embolisation for unresectable hepatocellular carcinoma. Cochrane Database Syst Rev 2011;(3):CD004787.

14. Llovet JM, Real MI, Montaña X, Planas R, Coll S, Aponte J, Ayuso C, et al.; Barcelona Liver Cancer Group. Arterial embolisation or chemoembolisation versus symptomatic treatment in patients with unresectable hepatocellular carcinoma: a randomised controlled trial. Lancet 2002;359(9319):1734-9.

15. Katsanos K, Kitrou P, Spiliopoulos S, Maroulis I, Petsas T, Karnabatidis D. Comparative effectiveness of different transarterial embolization therapies alone or in combination with local ablative or adjuvant systemic treatments for unresectable hepatocellular carcinoma: A network meta-analysis of randomized controlled trials. PLoS One 2017;12(9):e0184597.

16. Kirstein MM, Voigtländer T, Schweitzer N, Hinrichs JB, Marquardt J, Wörns MA, et al. Transarterial chemoembolization versus sorafenib in patients with 
hepatocellular carcinoma and extrahepatic disease. United European Gastroenterol J 2018;6(2):238-46.

17. Helal Tel A, Radwan NA, Shaker M. Extrahepatic metastases as initial manifestations of hepatocellular carcinoma: an Egyptian experience. Diagn Pathol 2015;10:82.

18. Yan L, Jakate S, Reddy V, Gattuso P. Metastatic hepatocellular carcinoma diagnosed by fine needle aspiration: A clinical and cytologic study. Diagn Cytopathol 2018;46(6):495-500.

19. Wang S, Wang A, Lin J, Xie Y, Wu L, Huang H, et al. Brain metastases from hepatocellular carcinoma: recent advances and future avenues. Oncotarget 2017;8(15):25814-29.

20. Ogino A, Hirai T, Serizawa T, Yoshino A. Clinical features of brain metastases from hepatocellular carcinoma using gamma knife surgery. Acta Neurochir (Wien) 2018;160(5):997-1003.
21. Kamimura K, Kobayashi Y, Takahashi Y, Abe H, Kumaki D, Yokoo T, et al. Tumor markers for early diagnosis for brain metastasis of hepatocellular carcinoma: A case series and literature review for effective loco-regional treatment. Cancer Biol Ther 2017;18(2):79-84.

22.Zhu AX, Park JO, Ryoo BY, Yen CJ, Poon R, Pastorelli $\mathrm{D}$, et al.; REACH Trial Investigators. Ramucirumab versus placebo as second-line treatment in patients with advanced hepatocellular carcinoma following first-line therapy with sorafenib (REACH): a randomised, double-blind, multicentre, phase 3 trial. Lancet Oncol 2015;16(7):859-70.

23. El-Khoueiry AB, Sangro B, Yau T, Crocenzi TS, Kudo $\mathrm{M}, \mathrm{Hsu} \mathrm{C}$, et al. Nivolumab in patients with advanced hepatocellular carcinoma (CheckMate 040): an openlabel, non-comparative, phase $1 / 2$ dose escalation and expansion trial. Lancet 2017;389(10088):2492-502. 